\title{
Global inhibition and midcourse corrections in speeded aiming
}

\author{
Erica L. Wohldmann, Alice F. Healy, \\ AND LyLE E. Bourne, JR. \\ University of Colorado, Boulder, Colorado
}

\begin{abstract}
When some perceptual-motor relationships are reversed, participants might adopt a global inhibition strategy that replaces all normal movements with reversed movements. In two experiments, participants practiced moving a cursor from a start position to target locations. In a perceptual-motor reversal condition, in which horizontal but not vertical movements were reversed, participants were trained to move only to certain locations. Testing involved moving to all locations under the same reversal condition. Training on a subset of locations yielded partial transfer to untrained locations. These results support a global inhibition hypothesis modified to include both midcourse corrective movements and training specificity.
\end{abstract}

To what extent is learning generalizable, extending beyond practiced items or specific task training requirements? Research exploring issues of transfer often has shown strong specificity of training, and various theoretical explanations have been proposed to account for this specificity. Thorndike (1906), possibly the first psychologist to identify this problem, formulated a theory of identical elements that suggests that transfer can be expected only to the degree that conditions of training and testing overlap in their elements. For Thorndike, the elements were stimulusresponse connections. More recently, Singley and Anderson (1989) took a computational approach to explaining specificity by the use of identical elements, which were production rules. Rickard and Bourne $(1995,1996)$ used similar ideas to account for the training of basic arithmetic skills, using operands and operators as elements. Numerous related hypotheses have been put forth to explicate the conditions under which transfer might be expected, including, for example, those involving encoding specificity (Tulving \& Thomson, 1973), transfer-appropriate processing (Morris, Bransford, \& Franks, 1977; Roediger, Weldon, \& Challis, 1989), procedural reinstatement (Healy \& Bourne, 1995; Healy, Wohldmann, \& Bourne, 2005), and sensorimotor representations underlying movement learning (Proteau, Marteniuk, \& Lévesque, 1992).

One way to reduce training specificity and to promote transfer might be to introduce variability of practice into the learning situation (see, e.g., Schmidt \& Bjork, 1992). The advantage for variable practice schedules was first predicted by Schmidt (1975), who formulated a hypothesis about variability of practice in terms of a schema theory. According to this theory, practice variability, by incorporating a large range of task elements, should promote the development and use of schemata (i.e., rules) that relate the general requirements of a task to the mental representation of the necessary motor movements (i.e., to a generalized motor program; for reviews, see Shea \& Wulf, 2005, and Van Rossum, 1990). The advantages of variable practice were first found in discrete motor tasks, but have since been observed in other types of tasks, including a continuous, feedback-regulated tracking task (Wulf \& Schmidt, 1997) and cognitive tasks (Schmidt \& Bjork, 1992).

Training variability effects might be limited to particular circumstances, however. Healy, Wohldmann, Sutton, and Bourne (2006) provided strong support for training specificity and no support for variability of practice on transfer of speeded-aiming movements in a handeye coordination task (Bourne, Healy, Pauli, Parker, \& Birbaumer, 2005). The task used by Healy et al. (2006) involved finding and moving to one of eight specified target locations that were arranged around the circumference of what looked like a clock face, but with fewer digits (see Figure 1). In addition to the normal condition, several types of perceptual-motor reversal conditions were examined that did not require the learning of new movements, but did require the learning of new stimulusresponse (cursor-mouse) relationships or rules. Specifically, in the vertical reversal condition, upward movements of the mouse produced downward movements of the cursor, and downward mouse movements produced upward cursor movements, but the horizontal movements remained normal. Similarly, in the horizontal reversal condition, horizontal movements of the mouse were reversed, but vertical movements remained normal. In the combined reversal condition, both vertical and horizontal movements were reversed. Healy et al. (2006) examined the effects of training in one or multiple reversal conditions on retention and transfer following a 1-week delay. 
8

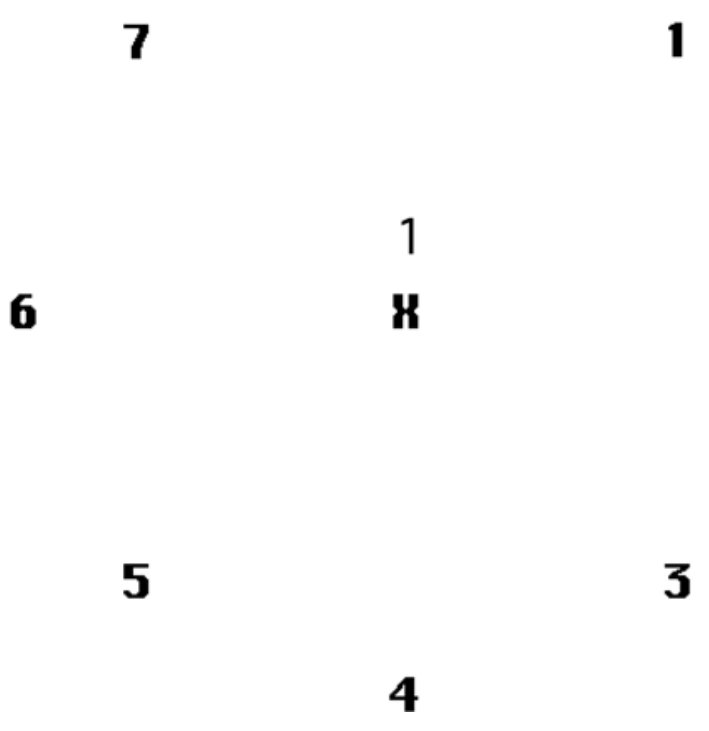

Figure 1. Clock face stimulus display. The target in the display shown is 1 .

Although the perceptual-motor reversals were difficult at the outset, performance improved across trial blocks. Participants showed no forgetting across the delay when the same reversal condition was used during both training and testing. However, little or no transfer was evident when the reversal condition was changed between training and testing, except for a small amount of positive transfer when participants practiced either the vertical or horizontal reversal during training and switched to the combined reversal during testing (i.e., part-whole transfer). Moreover, practice with multiple reversals during training did not promote subsequent transfer to a different reversal, providing no support for the hypothesized advantages of variability of practice.

To account for the severe specificity they observed, Healy et al. (2006) proposed that participants, recognizing that use of the mouse involved perceptual-motor incompatibilities, adopted a global inhibition strategy. According to the global inhibition hypothesis, mouse reversals require the inhibition (i.e., suppression) of normal mouse movements, applied globally to all normal movements. However, when the mouse is reversed horizontally but not vertically, or vice versa, in only one dimension, an additional step is required to disinhibit responses on the nonreversed dimension. Counterintuitively, this added step should result in slower movement times to the targets located along the nonreversed dimension than to those along the reversed dimension, a result that was found by Healy et al. (2006). The global inhibition strategy can also explain the finding of some degree of positive part-whole transfer (i.e., horizontal-to-combined or vertical-to-combined) because the global strategy, acquired during training, would apply directly to the transfer task, and there would be no need for disinhibition during testing. In addition, the global in- hibition strategy can explain the finding of interference or no transfer in the part-part (i.e., vertical-to-horizontal or horizontal-to-vertical) or whole-part (i.e., combined-tohorizontal or combined-to-vertical) directions. For partpart transfer, the inhibited and noninhibited dimensions must be exchanged between training and transfer, and for whole-part transfer, one of the previously inhibited dimensions must be disinhibited during transfer.

Healy et al. (2006) also considered an alternative hypothesis that involved midcourse corrective movements, which might account for the slower movement times along a nonreversed dimension than along a reversed dimension in the horizontal and vertical conditions. According to this hypothesis, when participants are required to make normal movements along a nonreversed dimension in a condition requiring reversed movements along only a single dimension, some corrective movements based on visual feedback might be required to get the cursor to land on the target, beyond any corrections that would be needed following entry into the wrong digit. That is, participants might start with a nonreversed movement and then notice that the produced movement went in a slightly wrong direction, so they correct their movement online. Such corrections must be made in the context of the reversal that is in effect. Thus, it could be that difficulty moving along the nonreversed dimension is not due entirely to inhibition of normal movements but instead is caused in part by the necessity to combine the normal movements with midcourse corrective movements. Unlike global inhibition, though, midcourse correction does not explain the finding of positive part-whole transfer and negative or no part-part and whole-part transfer.

The present study was conducted as a further examination of training specificity, of training variability effects, and of the global inhibition and midcourse correction hypotheses. Unlike in Healy et al. (2006), in the present study, only a brief rest period was interpolated between training and testing, thus eliminating the possible influence of normal practice during the 1-week retention interval. Also unlike the earlier study, we report a measure of initiation time, which is reaction time, or the time to leave the cursor start position after the onset of a target, as a supplement to movement time, which is the time to arrive at the target location on the circumference of the clock face after leaving the start position (see Bourne et al., 2005, for a discussion and comparison of these two measures and Henry, 1961, for a demonstration of the independence of these measures with respect to individual differences). Movement time should reflect the motor component of response execution, whereas initiation time should reflect the target-finding component of response execution, as well as any movement planning or programming (see, e.g., Rosenbaum, 1980, for a discussion of movement programming reflected in initiation time, and Elliott \& Lee, 1995, and Khan, Lawrence, Buckolz, \& Franks, 2006, for a consideration of the movementplanning or programming process in manual aiming). We expect that global inhibition and the disinhibition along specific dimensions should affect both initiation time and movement time, because these processes should be involved in movement planning (initiation time) as well 
as in the movement itself (movement time). In contrast, midcourse corrections should affect movement time but not initiation time, because these corrections necessarily occur during the course of movement itself, after planning is completed. Thus, a comparison of these two measures might help differentiate the effects of global inhibition and midcourse correction on performance.

\section{EXPERIMENT 1}

In Experiment 1, we explored the effects of limiting the training to a subset of targets on subsequent movement times to untrained targets in a single reversal condition. All participants were trained and tested in the horizontal reversal condition, but participants practiced moving to targets located on either the horizontal (2 and 6 ) or the vertical (4 and 8) dimension, as well as on one of two diagonal dimensions (1 and 5, or 3 and 7) (see Figure 1). Thus, participants always practiced four of the eight targets during training and, following a 5-min break, were tested on all eight targets. According to the global inhibition hypothesis, positive transfer to the untrained targets along the diagonal axis should be evident, because both the trained and untrained targets along a diagonal axis demand that horizontal movements be inhibited and vertical movements be disinhibited in the same way. The global inhibition hypothesis would, thus, predict no differences in transfer for trained and untrained movements. The midcourse correction hypothesis asserts that bilateral corrective movements might be needed to reach the desired target. These corrective movements teach participants about the configuration of inhibition and disinhibition that is required with the reversal to reach the target locations not included in the training set. For example, in the horizontal reversal condition, a participant who applies midcourse corrective movements to reach the target 8 should, in effect, acquire some knowledge about and practice with the movements required to reach the targets on the untrained diagonal axis. Therefore, both the global inhibition and midcourse correction strategies should yield perfect transfer to the targets located along the untrained diagonal axis, so there would be no advantage for the trained over the untrained movements along the diagonal axes. Finding an advantage for trained over untrained movements would, thus, challenge both the global inhibition and midcourse correction hypotheses, suggesting that they need to be amended or supplemented by a specificity-of-training principle. Indeed using a similar paradigm, Krakauer, Pine, Ghilardi, and Ghez (2000) found specificity in a study involving directional reaching movements under a screen cursor rotation. These findings imply that the skill learned in our clock face paradigm should also depend on the particular target locations practiced and on strategies such as global inhibition and midcourse correction.

\section{Method}

Participants. Twenty-four undergraduate students from the University of Colorado at Boulder participated for credit in an introductory psychology course. Participants were assigned by fixed rotation to one of four training conditions, with 6 participants in each condition.
Design. This experiment had a $4 \times 2 \times 12$ mixed factorial design, including training condition-horizontal-diagonal 1/5 (HD1), horizontal-diagonal 3/7 (HD2), vertical-diagonal 1/5 (VD1), and vertical-diagonal 3/7 (VD2) - as a between-subjects variable, and session half (training, testing) and block (1-12) as within-subjects variables. To examine transfer, focused analyses on testing alone were conducted that were restricted to either the diagonal or the nondiagonal (horizontal and vertical) axes with target type (old, new) included as a factor. The dependent variables were movement and initiation times.

Apparatus, Materials, and Procedure. The task involved finding and moving to a single target location on each trial by moving the cursor quickly and accurately from a center fixation point to the correct location on the circumference of the clock face using a mouse that was reversed horizontally but not vertically. Participants were individually tested in quiet rooms on iMac G3 15-in. computers. They sat (at approximately a $0.5-\mathrm{m}$ distance) in front of a computer screen that displayed a clock face consisting of the center fixation point $X$ surrounded by a circle of equally spaced digits, in the order $1-8$, with the target for a given trial always indicated directly above the fixation point (see Figure 1). The diameter of the clock face display was approximately $20 \mathrm{~cm}$. The center fixation and the target digit were each surrounded by an invisible circle approximately $1.6 \mathrm{~cm}$ in diameter. The participants were required to place the cursor within the center circle to initiate a trial and to place it within the target circle to conclude a trial. As soon as the cursor reached the target circle, the circle surrounding the target digit was highlighted. Participants were required to enter the circle for the target digit on every trial. The next trial would not begin until the participant reached the correct target digit, so no errors were scored. Participants could take a brief break between trials by delaying their entry into the center circle, at which point the next target digit was displayed immediately. Participants practiced 200 trials in each session half, and each session half was divided into 12 blocks of 16 trials, followed by a final set of 8 trials. The final set of trials was omitted from the analyses because not all targets were included; thus, only the first 192 trials were included in the analyses.

During training, participants were given only four of the eight targets to practice, with four trials with each target in each block. Specifically, participants in the HD1 and HD2 conditions practiced moving along the horizontal axis $(2,6)$ and on one diagonal axis. Participants in the HD1 condition practiced as well along one diagonal axis $(1,5)$, and participants in the HD2 condition practiced along the opposite diagonal axis $(3,7)$. Similarly, participants in the VD1 and VD2 conditions practiced moving along the vertical axis $(4,8)$; those in the VD1 condition practiced along one diagonal axis $(1,5)$, and those in the VD2 condition practiced moving along the opposite diagonal axis $(3,7)$. During testing, there were two trials with each of the eight targets arranged in a pseudorandom order in each block. All participants were given the trials in the same order. Between training and testing, participants were given a 5-min break in which they were asked to rest quietly. At the start of training, a brief demonstration of the required procedures was presented as an animation on the computer screen, and participants were told, "First place the cursor on the $\mathrm{X}$ in the center of the screen. Next, wait for the target digit to appear above the X. Then move the cursor to the correct target digit. Last, move the cursor back to the X in the center of the screen." Participants were given no instructions indicating that there would be any mouse reversal, and to ensure that all learning would occur during the experimental trials, they were given no practice trials.

The monitor resolution was set at its largest value $(640 \times 480)$, so the display filled the screen. The computer program for reversing mouse-cursor movements was written in HyperCard. This program did not capture movement trajectories (including entries into incorrect digit locations); thus, no kinematics analysis was possible. To ensure that participants used the mouse in its upright position, tape was applied to the mouse cord approximately $15 \mathrm{~cm}$ above the top of the mouse. Participants were monitored through windows on the testing room doors throughout the experiment. 


\section{Results}

For all analyses, we used an $\alpha$ level of .05 as a criterion of statistical significance. For the analysis of transfer, we compared both movement and initiation times on the trained (old) diagonal axis with those on the untrained (new) diagonal axis during testing. A similar analysis was conducted on the old and new nondiagonal movements (i.e., movements along either the vertical or horizontal axis). However, the results of the nondiagonal movements are not reported here, because movement times along the horizontal axis are always fastest (presumably because of the required disinhibition along the vertical axis), making the transfer results difficult to interpret. The results of the analyses on movement times for the diagonal axes including the factors of training condition, target type (old, new), and block (1-12) are shown in the top panel of Figure 2 (see the lines with unfilled symbols) as a function of target type and block. It took less time to move to old targets than to new targets (compare the solid and dashed lines) $\left[F(1,20)=37.41, M S_{\mathrm{e}}=.136\right]$, but the difference

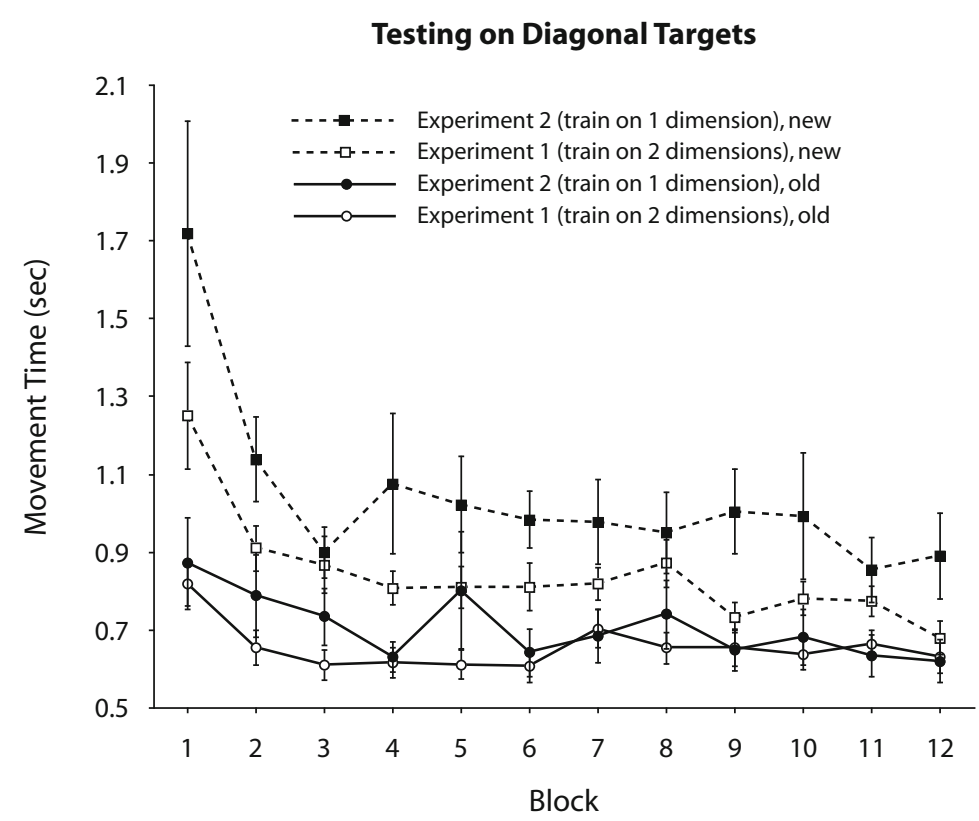

Testing on Diagonal Targets

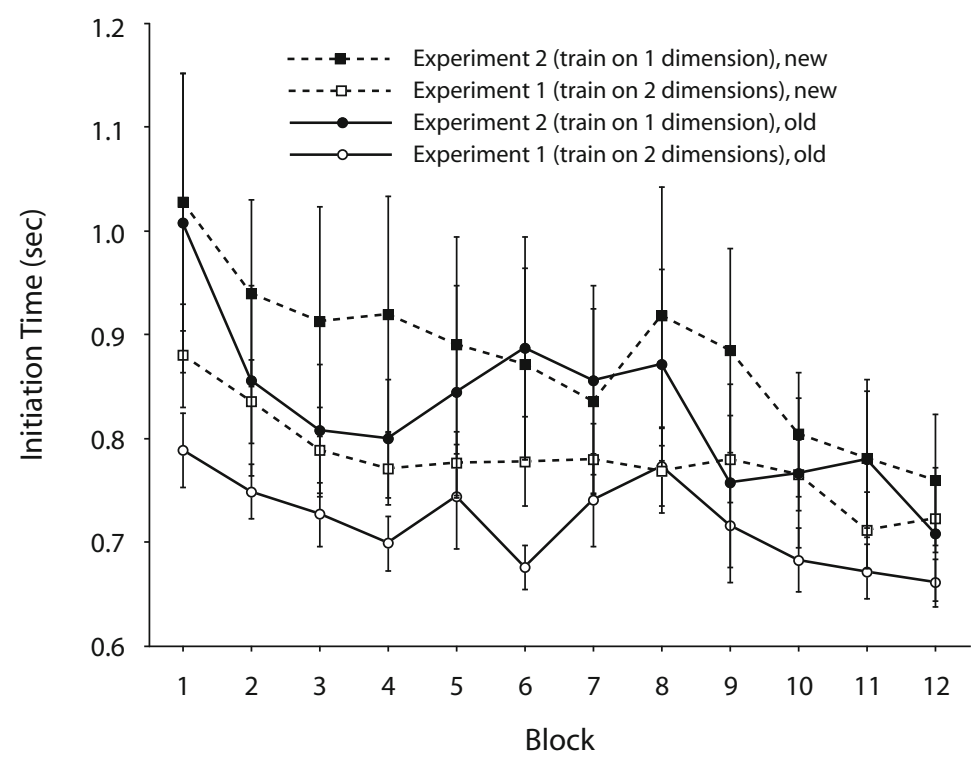

Figure 2. Mean movement time (top panel) and initiation time (bottom panel) (in seconds), during testing for diagonal movements as a function of experiment, target type (old, new), and block (1-12) in Experiments 1 and 2. Error bars represent positive and negative standard errors of the mean. 
between movement times for old and new targets diminished across blocks; the interaction of target type and block was significant $\left[F(11,220)=3.25, M S_{\mathrm{e}}=.039\right]$. Participants became faster overall across blocks of testing; the main effect of block was significant $[F(11,220)=8.96$, $\left.M S_{\mathrm{e}}=.050\right]$.

In terms of initiation time, performance generally improved across blocks $\left[F(11,220)=6.21, M S_{\mathrm{e}}=.013\right]$ (see the lines with unfilled symbols in the bottom panel of Figure 2). As was found for movement times, it took less time to initiate movements to old targets than to new targets (compare the solid and dashed lines) $[F(1,20)=26.61$, $\left.M S_{\mathrm{e}}=.020\right]$. However, the difference in initiation times between old and new targets depended on training condition; the interaction of target type and training condition was significant $\left[F(3,20)=6.85, M S_{\mathrm{e}}=.020\right]$. Specifically, participants in the HD2 condition initiated movements to new targets $(0.706 \mathrm{sec})$ a bit faster than to old targets $(0.712 \mathrm{sec})$, but a larger difference in the opposite direction was found for all other conditions (HD1, old = $0.795 \mathrm{sec}$, new $=0.921 \mathrm{sec} ; \mathrm{VD} 1$, old $=0.637 \mathrm{sec}$, new $=$ $0.735 \mathrm{sec} ; \mathrm{VD} 2$, old $=0.733 \mathrm{sec}$, new $=0.757 \mathrm{sec})$, with the pattern somewhat variable across blocks $[F(33,220)=$ $\left.1.66, M S_{\mathrm{e}}=.008\right]$.

For both movement and initiation times, we conducted additional analyses of transfer. Specifically, response times on the untrained diagonal targets in the first five blocks of testing for a given group of participants were compared with response times on the same diagonal targets in the first five blocks of training by the other groups of participants who were given these targets during training. This comparison was made using between-subjects $t$ tests. A mean difference between training and testing of 0 would indicate no transfer, whereas a positive difference would indicate positive transfer and a negative difference would indicate negative transfer. By this analysis, for movement time, significant positive transfer was found in all but the HD1 training condition $[t(16)=4.57$ for HD2, 4.57 for VD1, and 2.51 for VD2] (see Table 1 for the mean differences between training and testing). Positive transfer for the HD1 condition was only marginally significant $[t(16)=1.88, p=.078]$. For initiation time, significant

Table 1

Mean Differences Between First Five Blocks of Training and First Five Blocks of Testing in Movement and Initiation Time for Each Training Condition of Experiments 1 and 2

\begin{tabular}{ccc}
\hline Training & & \\
Condition & Movement Time & Initiation Time \\
\hline \multicolumn{3}{c}{ Experiment 1} \\
HD1 & 0.416 & -0.013 \\
HD2 & $1.035^{*}$ & $-0.220^{*}$ \\
VD1 & $0.908^{*}$ & -0.016 \\
VD2 & $0.530^{*}$ & -0.025 \\
& Experiment & \\
H & 0.313 & -0.117 \\
V & $0.560^{*}$ & -0.007 \\
D1 & 0.246 & 0.076 \\
D2 & 0.284 & -0.225 \\
\hline
\end{tabular}

${ }^{*} p<.05$. negative transfer was found in the HD2 condition $[t(16)$ $=2.90$ ], with no other conditions showing any significant transfer (see Table 1).

\section{Discussion}

During testing, the fact that initiation and movement times were faster to those diagonal targets practiced during training than to the untrained diagonal targets indicated specificity. In contrast, movement times were faster to the untrained targets during the first five blocks of testing than to those same targets during the first five blocks of training, implying some degree of generalizability of training. A different pattern was found for initiation times, with significant negative transfer for the HD2 training condition, which reflected slower initiation times for the untrained diagonal targets after training than for the same diagonal targets at the start of training. This finding implies that the movement planning (and/ or the target finding) during initiation time is specific to the trained targets, but that the execution that occurs during movement time is an aspect of the skill that is generalizable. Finding different patterns for movement and initiation times is also consistent with the midcourse correction hypothesis, but not with the global inhibition hypothesis on its own.

\section{EXPERIMENT 2}

All participants in Experiment 2 were trained and tested in the horizontal reversal condition, with training limited to a single axis - vertical (4 and 8), horizontal (2 and 6), or diagonal (1 and 5, or 3 and 7) (see Figure 1) — but with testing involving all targets. If participants adopt a global inhibition strategy, transfer to the untrained diagonal axis should be evident when training involves moving along a diagonal axis. On the other hand, participants should not learn either to inhibit or to disinhibit responses when training is restricted to the vertical axis, in which case no transfer to the untrained target locations would be expected. Finding positive transfer to the untrained diagonal axes if training involved practice with the target locations along the vertical axis would suggest strongly that global inhibition alone is an inadequate account, because transfer would mean that participants were making midcourse corrective movements in the horizontal direction to reach the locations along the vertical axis. Transfer from the horizontal axis to the untrained diagonal axes would likewise suggest that midcourse corrective movements (which in this case involve some disinhibition of vertical movements) were being applied to reach the trained target locations and, thus, would provide additional support for supplementing global inhibition with midcourse correction.

In contrast to the results of others (Schmidt \& Bjork, 1992; Wulf \& Schmidt, 1997), Healy et al. (2006) found no evidence in support of the variability-of-practice principle. In Experiment 2, we test this principle in a different way. Specifically, Healy et al. (2006) required participants to learn one type or several types of perceptualmotor reversal during training and then to apply what they had learned to a different type of perceptual-motor 
reversal during testing. This manipulation of variability is a between-task manipulation (i.e., between different reprogrammed mice, which might require different generalized motor programs), as opposed to a within-task manipulation (i.e., within a single reprogrammed mouse, which presumably uses a single generalized motor pattern). Typically, the within-task manipulation has been used to explore the effects of practice variability on transfer of learning (see, e.g., Kerr \& Booth, 1978, who varied the target distance at which bean bags were tossed; but see, e.g., Seidler, 2004, for the advantage of variable practice with a between-task manipulation involving different joystick-aiming tasks). A within-task manipulation of variability was used in the present study by requiring participants to apply a learned perceptual-motor reversal to untrained movements, or target locations. Test performance in Experiment 1, which involved training on four targets and testing on all eight, was compared with test performance in Experiment 2, which involved training on only two targets and testing on all eight (see Krakauer et al., 2000, for a similar manipulation of variability of practice that showed the advantage of multiple targets for directional reaching movements under screen cursor rotation). If variability of practice promotes transfer, we would expect to find more transfer in Experiment 1, which involved practice with four different target locations, than in Experiment 2, which involved practice with only two locations.

\section{Method}

Participants. Twenty-four undergraduate students from the University of Colorado at Boulder participated for credit in an introductory psychology course. Again, participants were assigned by fixed rotation to one of the four training conditions, with 6 participants in each condition.

Design. As in Experiment 1, the design for Experiment 2 was a $4 \times 2 \times 12$ mixed factorial, including training condition-horizontal (H), vertical (V), diagonal 1/5 (D1), diagonal 3/7 (D2) as a between-subjects variable and session half (training, testing) and block (1-12) as within-subjects variables. Again, to examine transfer, focused analyses on testing alone were conducted that were restricted to the diagonal axes, with target type (old, new) included as a factor, and the dependent variables were the movement and initiation times.

Apparatus, Materials, and Procedure. The procedure used for Experiment 1 was used for Experiment 2, with one exception. During training, participants in each condition were given only two of the eight targets to practice. Specifically, participants in the H condition practiced moving along the horizontal axis $(2,6)$, those in the $\mathrm{V}$ condition practiced moving along the vertical axis $(4,8)$, those in the D1 condition practiced moving along one of the diagonal axes $(1,5)$, and those in the D2 condition practiced moving along the opposite diagonal axis $(3,7)$.

\section{Results}

Transfer. As in Experiment 1, to assess transfer, both movement and initiation times on the trained (old) diagonal axis were compared with those on the untrained (new) diagonal axis during testing, including the factors of training condition, target type, and block. Only the participants trained with a diagonal axis were included in this analysis. Again, movement times were faster to old than to new targets $\left[F(1,10)=32.92, M S_{\mathrm{e}}=.247\right]$ (compare the solid and dashed lines with the filled symbols in the top panel of Figure 2). In addition, the difference between movement times for old and new targets became smaller across blocks $\left[F(11,110)=2.95, M S_{\mathrm{e}}=.066\right]$, and participants' performance improved overall across blocks of testing $\left[F(11,110)=5.64, M S_{\mathrm{e}}=.090\right]$.

With respect to initiation times, performance generally improved across blocks $\left[F(11,110)=5.42, M S_{\mathrm{e}}=.023\right]$. Also, participants were faster to initiate movements to old targets than to new targets $\left[F(1,10)=8.45, M S_{\mathrm{e}}=.021\right]$ (compare the solid and dashed lines with the filled symbols in the bottom panel of Figure 2). However, this difference between old and new targets was significantly greater for participants in the D1, condition (old $=0.729 \mathrm{sec}$, new $=0.820 \mathrm{sec}$ ) than for those in the D2 condition (old $=0.929 \mathrm{sec}$, new $=0.937 \mathrm{sec})$; the interaction of training condition and target type was significant $[F(1,10)=6.02$, $\left.M S_{\mathrm{e}}=.021\right]$.

As in Experiment 1, for both movement and initiation times, we conducted additional analyses of transfer to the untrained diagonal axis (or axes for the $\mathrm{H}$ and $\mathrm{V}$ conditions). (See Table 1 for the mean difference between the first five blocks of training and the first five blocks of testing.) The analyses on movement times revealed significant transfer to the diagonal targets in the case when training involved moving along the nonreversed dimension (V). That is, movement time on the untrained diagonal axes in the first five blocks of testing by the $\mathrm{V}$ training condition was significantly shorter than movement time for diagonal targets in the first five blocks of training by the D1 and D2 training conditions, implying positive transfer in this case $[t(16)=3.08]$. For initiation time, no significant transfer was found in any condition (see Table 1).

Variability of practice. To examine the variabilityof-practice hypothesis, we compared test performance on diagonal targets in Experiment 1, which involved training with two types of movement (along two axes), against test performance on diagonal targets in Experiment 2, which involved training with only one type of movement (along a single axis). Specifically, we conducted analyses on both movement and initiation times for diagonal targets during testing with the factors of target type (old, new), experiment $(1,2)$, and block (1-12). These analyses were limited to participants who trained with one diagonal and tested with both diagonals; thus, all participants in Experiment 1 were included in the analysis, but only half of the participants in Experiment 2 were included.

The results of the comparison are summarized in Figure 2, including movement time in the top panel and initiation time in the bottom panel. The only significant effect involving the factor of experiment in these analyses was a significant interaction between target type and experiment for movement time $\left[F(1,34)=5.97, M S_{\mathrm{e}}=.177\right]$, showing a larger advantage for Experiment 1 relative to Experiment 2 (i.e., for training with four as opposed to two locations) for new targets than for old targets. Thus, in this case, variability of practice does seem to have enhanced transfer of training to new targets but only with respect to movement time, not initiation time. 


\section{Discussion}

Both initiation and movement times were faster at test to the trained targets than to the untrained targets, demonstrating specificity of training. Demonstrating generalizability of training, movement times were faster to the untrained targets located along the diagonal axes in the first five blocks of testing than in the first five blocks of training, but significantly so only when training involved moving along the vertical (nonreversed) dimension. That is, transfer to untrained diagonal movements was actually significant for the $\mathrm{V}$ condition (in which no diagonal movements had been trained) but not significant for the D1 and D2 conditions (in which the opposite diagonal movement had been trained). This finding was unanticipated and cannot be explained by the global inhibition hypothesis; it clearly suggests that, during training, participants must have applied midcourse corrective movements to reach the desired locations. The fact that this result was obtained for movement time but not for initiation time is also supportive of the midcourse correction hypothesis, which (unlike the global inhibition hypothesis) predicts a different pattern of results for the two measures. Finally, the advantage for trained relative to untrained targets suggests that participants learned some specific movement patterns for diagonal movements beyond those implicated by either the global inhibition hypothesis or the midcourse correction hypothesis, and those specific patterns apply to one diagonal movement but not to another. The advantage for trained over untrained movements was found for initiation time as well as movement time, suggesting that the advantage may reflect movement planning or target finding as well as the actual movement processes themselves.

Contrary to the previous findings by Healy et al. (2006, Experiment 3), the present study indicated that, in some cases, variable training can promote transfer. Experiment 1, which involved training with four target locations, yielded more transfer to the untrained target locations than was seen in Experiment 2, which involved training with only two target locations; however, this advantage was found only for new targets and only for the measure of movement time.

\section{GENERAL DISCUSSION}

The present study was conducted to examine circumstances under which learning can generalize beyond the specific conditions practiced during training. On the basis of earlier research and theory, including the theory of identical elements (e.g., Thorndike, 1906), encoding specificity (e.g., Tulving \& Thomson, 1973), transfer-appropriate processing (e.g., Morris et al., 1977), procedural reinstatement (e.g., Healy et al., 2005), and sensorimotor representations underlying movement learning (e.g., Proteau et al., 1992), severe specificity of training was expected. Indeed, in an earlier study using the same computer-displayed perceptual-motor task, Healy et al. (2006) found little or no transfer when the conditions for testing did not match those for training.

Even stronger specificity was found in the present study. In both experiments, diagonal initiation and move- ment times during testing were significantly faster to the trained than to the untrained targets, implying specific training effects that apply both to movement planning and to actual movement, and perhaps to target finding. These specific training effects resemble those found by Keetch, Schmidt, Lee, and Young (2005) for expert basketball players taking set shots at the foul line. Keetch et al. referred to these as "especial skills," although the specific training effects in our case, unlike those of Keetch et al., occurred with novices. However, we also found some positive transfer, so that there was some degree of generality of the learned skill, as well as some degree of specificity. In particular, diagonal movement times to the untrained items during the first five blocks of testing were significantly shorter than those to the same items when given to other participants during the first five blocks of training, both when training involved two dimensions (in Experiment 1) and when it involved moving along the nonreversed dimension (in Experiment 2). The significant transfer in Experiment 2 was found only in the $\mathrm{V}$ condition. If participants in the $\mathrm{V}$ condition were moving directly to the targets presented during training without making bilateral midcourse corrective movements, then, according to the global inhibition hypothesis, no transfer would be expected, because only movements along the horizontal dimension were reversed. However, if participants in the $\mathrm{V}$ condition were learning also to make midcourse corrective movements to reach the targets presented during training, then those corrective movements should teach them something about the configuration of inhibition and disinhibition required to reach the targets located along the diagonal axes. In this case, transfer to those axes would be expected, and this transfer should show up in faster movement times but not in faster initiation times, which was indeed the pattern observed. Together, the results suggest that the strategy employed by participants is more complex than can be explained by an unaugmented global inhibition hypothesis. A full explanation requires a provision for specificity of training, as well as for midcourse correction (presumably beyond any correction needed as a result of entry into the wrong digit). Stronger support for midcourse correction would require kinematics data, but this type of data was not collected in the present study.

Healy et al. (2006) found no variability-of-practice effect. However, numerous other studies have shown that introducing variable practice during training facilitates retention and transfer (e.g., Krakauer et al., 2000; Schmidt \& Bjork, 1992; Shea \& Wulf, 2005; Wulf \& Schmidt, 1997). Thus, in addition to testing the global inhibition and midcourse correction hypotheses, Experiments 1 and 2 were aimed to determine whether a different kind of variable practice schedule would promote more transfer than would a constant practice schedule. In the present study, we found some evidence to suggest an advantage for variability of practice, in that practice with four targets (Experiment 1) led to greater transfer of movement times to the untrained diagonal targets than did practice with only two targets (Experiment 2). The discrepancy be- 
tween the present study and the earlier one by Healy et al. (2006) seems likely to be related to the type of variability examined. In the present study, variability was achieved in terms of different target locations with a fixed reversed mouse (a within-task manipulation involving a single generalized motor pattern), whereas in the earlier study, variability was achieved in terms of different mouse reversals with fixed target locations (a between-task manipulation involving different generalized motor patterns). If each reversal condition is assumed to involve a distinct configuration of responses (i.e., a distinct generalized motor program), practicing with multiple reversal conditions might not strengthen any one configuration, but practicing with multiple target locations within a single reversal condition should strengthen that configuration.

In general, the results of the present line of research support four different but interrelated conclusions about the relationship between training and transfer in speeded aiming, with and without perceptual-motor reversals. First, when they encounter a reversal of normal perceptual-motor relationships along a given dimension, participants adopt a global inhibition strategy, inhibiting all normal movements along all dimensions, but then disinhibiting movements on the nonreversed dimension. Second, participants engage in midcourse corrective movements on the basis of visual feedback to reach the desired target, even when moving along a nonreversed dimension. Third, in the course of training, participants learning a given reversal condition acquire highly specific movement tactics that apply to each target location. Fourth, participants' movements benefit from practice variability if that variability involves different targets within a given reversal condition, but not if that variability involves different reversal conditions. Although these conclusions were drawn from a particular task involving stimulus-response incompatibility in speeded aiming, they are likely to apply more generally to any tasks that require cognitive control of motor responses.

\section{AUTHOR NOTE}

This research was supported in part by Army Research Institute Contract DASW01-03-K-0002 and Army Research Office Grant W9112NF-05-1-0153 to the University of Colorado. We are grateful to Martina Rieger and Howard Zelaznik for helpful comments about this research. Please address correspondence to E. L. Wohldmann, Department of Psychology, California State University, 18111 Nordhoff St., Northridge, CA 91330-8255 (e-mail: erica.wohldmann@csun.edu).

\section{REFERENCES}

Bourne, L. E., Jr., Healy, A. F., Pauli, P., Parker, J. T., \& BirBAUMER, N. (2005). The influence of stimulus array on training of a speeded response. American Journal of Psychology, 118, 385-411.

Elliott, D., \& Lee, T. D. (1995). The role of target information on manual-aiming bias. Psychological Research, 58, 2-9.

Healy, A. F., \& Bourne, L. E., JR. (1995). Preface: Durability and specificity of knowledge and skills. In A. F. Healy \& L. E. Bourne, Jr. (Eds.), Learning and memory of knowledge and skills: Durability and specificity (pp. ix-xiv). Thousand Oaks, CA: Sage.

Healy, A. F., Wohldmann, E. L., \& Bourne, L. E., JR. (2005). The procedural reinstatement principle: Studies on training, retention, and transfer. In A. F. Healy (Ed.), Experimental cognitive psychology and its applications (pp. 59-71). Washington, DC: American Psychological Association.

Healy, A. F., Wohldmann, E. L., Sutton, E. M., \& Bourne, L. E., JR. (2006). Specificity effects in training and transfer of speeded responses. Journal of Experimental Psychology: Learning, Memory, \& Cognition, 32, 534-546.

Henry, F. M. (1961). Reaction time-movement time correlations. Perceptual \& Motor Skills, 12, 63-66.

Keetch, K. M., Schmidt, R. A., Lee, T. D., \& Young, D. E. (2005). Especial skills: Their emergence with massive amounts of practice. Journal of Experimental Psychology: Human Perception \& Performance, 31, 970-978.

Kerr, R., \& Bоотн, B. (1978). Specific and varied practice of motor skill. Perceptual \& Motor Skills, 46, 395-401.

Khan, M. A., Lawrence, G. P., Buckolz, E., \& Franks, I. M. (2006). Programming strategies for rapid aiming movements under simple and choice reaction time conditions. Quarterly Journal of Experimental Psychology, 59, 524-542.

Krakauer, J. W., Pine, Z. M., Ghilardi, M.-F., \& Ghez, C. (2000). Learning of visuomotor transformations for vectorial planning of reaching trajectories. Journal of Neuroscience, 20, 8916-8924.

Morris, C. D., Bransford, J. D., \& Franks, J. J. (1977). Levels of processing versus transfer appropriate processing. Journal of Verbal Learning \& Verbal Behavior, 16, 519-533.

Proteau, L., Marteniuk, R. G., \& Lévesque, L. (1992). A sensorimotor basis for motor learning: Evidence indicating specificity of practice. Quarterly Journal of Experimental Psychology, 44A, 557-575.

RickARD, T. C., \& Bourne, L. E., JR. (1995). An identical-elements model of basic arithmetic skills. In A. F. Healy \& L. E. Bourne, Jr. (Eds.), Learning and memory of knowledge and skills: Durability and specificity (pp. 255-281). Thousand Oaks, CA: Sage.

Rickard, T. C., \& Bourne, L. E., JR. (1996). Some tests of an identical elements model of basic arithmetic skills. Journal of Experimental Psychology: Learning, Memory, \& Cognition, 22, 1281-1295.

Roediger, H. L., III, Weldon, M. S., \& Challis, B. H. (1989). Explaining dissociations between implicit and explicit measures of retention: A processing account. In H. L. Roediger III \& F. I. M. Craik (Eds.), Varieties of memory and consciousness: Essays in honour of Endel Tulving (pp. 3-41). Hillsdale, NJ: Erlbaum.

Rosenbaum, D. A. (1980). Human movement initiation: Specification of arm, direction, and extent. Journal of Experimental Psychology: General, 109, 444-474.

ScHmidT, R. A. (1975). A schema theory of discrete motor skill learning. Psychological Review, 82, 225-260.

Schmidt, R. A., \& BJoRK, R. A. (1992). New conceptualizations of practice: Common principles in three paradigms suggest new concepts for training. Psychological Science, 3, 207-217.

SEIDLER, R. D. (2004). Multiple motor learning experiences enhance motor adaptability. Journal of Cognitive Neuroscience, 16, 65-73.

SheA, C. H., \& Wulf, G. (2005). Schema theory: A critical appraisal and reevaluation. Journal of Motor Behavior, 37, 85-101.

Singley, M. K., \& Anderson, J. R. (1989). The transfer of cognitive skill. Cambridge, MA: Harvard University Press.

THORNDIKE, E. L. (1906). The principles of teaching: Based on psychology. New York: A. G. Seiler.

Tulving, E., \& Thomson, D. M. (1973). Encoding specificity and retrieval processes in episodic memory. Psychological Review, 80, 352-373.

VAN Rossum, J. H. (1990). Schmidt's schema theory: The empirical base of the variability of practice hypothesis: A critical analysis. Human Movement Science, 9, 387-435.

Wulf, G., \& Schmidt, R. A. (1997). Variability of practice and implicit motor learning. Journal of Experimental Psychology: Learning, Memory, \& Cognition, 23, 987-1006.

(Manuscript received January 18, 2008; revision accepted for publication May 5, 2008.) 